\title{
Mechanical Properties of Hydrated Cement Paste: Development of Structure-property Relationships
}

\author{
Tewodros T. Ghebrab ${ }^{1)}$ and Parviz Soroushian²)
}

(Received September 10, 2009, Revised December 9, 2009, Accepted March 30, 2010)

\begin{abstract}
Theoretical models based on modern interpretations of the morphology and interactions of cement hydration products are developed for prediction of the mechanical properties of hydrated cement paste (hcp). The models are based on the emerging nanostructural vision of calcium silicate hydrate (C-S-H) morphology, and account for the intermolecular interactions between nano-scale calcium C-S-H particles. The models also incorporate the effects of capillary porosity and microcracking within hydrated cement paste. The intrinsic modulus of elasticity and tensile strength of hydrated cement paste are determined based on intermolecular interactions between C-S-H nano-particles. Modeling of fracture toughness indicates that frictional pull-out of the micro-scale calcium hydroxide $(\mathrm{CH})$ platelets makes major contributions to the fracture energy of hcp. A tensile strength model was developed for hcp based on the linear elastic fracture mechanics theories. The predicted theoretical models are in reasonable agreements with empirical models developed based on the experimental performance of hcp.
\end{abstract}

Keywords: mechanical properties, hydrated cement paste, molecular interactions, capillary porosity, calcium-silicate-hydrate.

\section{Introduction}

Hydrated cement paste (hcp) is a complex composite material with multiple phases at micro- and nano-scales. These features complicate development of structure-property relationships for hydrated cement paste. Several empirical structure-property relationships have been developed for hydrated cement paste based on laboratory experiments. ${ }^{1,2}$ Computational tools are also used toward modeling of hydrated cement paste.

The multi-phase nature of hydrated cement paste at different scales distinguishes it from technical ceramics. Unlike most ceramics, hydrated cement paste does not fracture in a brittle mode. It is heterogeneous at micro-scale, where capillary pores, large $\mathrm{CH}$ crystals and shrinkage microcracks are distributed randomly. The presence of these micro-defects produces stress concentrations which weaken the strength and stiffness of the material, but contribute to the fracture toughness of hcp through arrest and deflection of microcracks, formation of microcrack processing zones, and frictional energy dissipation during pull-out of $\mathrm{CH}$ crystals. These phenomena render hcp semi-brittle with some level of ductility and energy absorption capacity.

This paper presents analytical models for the mechanical performance of hydrated cement paste. The models incorporate intermolecular interactions between nano-scale C-S-H particles, effects of

\footnotetext{
${ }^{1)}$ Dept. of Engineering Technology, Texas Tech University, TX 79409-3107, USA. E-mail: ghebrabt@gmail.com.

${ }^{2)}$ Dept. Civil and Environmental Engineering, Michigan State University, MI 48824-1226, USA.E-mail: soroushi@egr.msu.edu. Copyright (c) 2010, Korea Concrete Institute. All rights reserved, including the making of copies without the written permission of the copyright proprietors.
}

micro-defects (capillary pores and microcracks), and frictional pull-out of CH micro-scale platelets. Modeling of C-S-H as nanoparticles revolve an evolving view of C-S-H morphology, ${ }^{6}$ compared to the traditional laminar vision of the C-S-H structure.

The models for hcp are developed in two steps. The first step focuses on compact hcp, and the second step incorporates the effects of micro-defects into the models developed for the compact hcp. The term "compact" is used in this paper to indicate hcp free from micro defects.

\section{Compact hydrated cement paste}

\subsection{Molecular interactions between C-S-H particles}

Mechanical models for compact hcp were first developed based on phase interactions at molecular level. These models were later modified by introducing the effects of micro-defects for application to hcp with micro-defects. This section focuses on compact hcp.

The interactions between nano-scale C-S-H particles play key roles in determining the mechanical properties of hcp. ${ }^{3} \mathrm{C}-\mathrm{S}-\mathrm{H}$ particles are non-crystalline hydration products with large surface areas which enhance their bonding to each other and to other particles within their reach through van der waals interactions. Therefore, C-S-H plays a predominant role in rendering hep its cementitious properties. ${ }^{4}$

Given the significant binding role of C-S-H particles, the first step in theoretical modeling focused on the morphology and structural arrangement of C-S-H particles in order to determine the bond strength between them. Research toward determination of the detailed morphology of C-S-H is still in progress. C-S-H has been likened by several researchers to Tobermorite mineral 
because of their similar compositions. ${ }^{5}$ Although C-S-H particles grow to have a size of approximately $60 \times 30 \times 5 \mathrm{~nm}^{3}$, they have been modeled as an agglomeration of unit particles making a spherical globule. ${ }^{6}$ This geometrical modeling was adopted in the research reported herein. An agglomeration of basic C-S-H units is assumed to make a spherical C-S-H particles (globule) with diameter ranging from 6 to $8 \mathrm{~nm}$ (Fig. 1). ${ }^{7}$ Due to the availability of water and space, the C-S-H particles which form at the outer part of hydrating cement grain have lower density than the ones which form at the inner part. ${ }^{6}$ A low density C-S-H is assumed to be an agglomeration of globules bonding to each other and having a gel porosity of about $28 \%$ (of the globule volume). ${ }^{6}$

The bonding between C-S-H globules involve van der waals interactions caused by weak physical electrostatic attractions between molecules in neighboring globules. The van der waals force per unit area $\left(F_{v d w}\right)$ between two flat surfaces separated by a distance $d$ can be expressed as follows: ${ }^{8}$

$$
F_{v d w}=\frac{3 h_{w}}{16 \pi^{2} d^{3}}
$$

where $h_{w}$ is the dielectric constant, approximately $10 \mathrm{eV}$ or 1.6022 $\times 10^{-18} \mathrm{~J}$ for C-S-H. ${ }^{8}$ This equation can be applied to C-S-H globules as far as an effective (circular) area is used with $\mathrm{d}$ taken as the minimum distance between two C-S-H globules ( 0.18 to $0.32 \mathrm{~nm}){ }^{4}$

Substituting the above values into Eq. (1) gives a van der waals bond strength between two adjacent C-S-H globules of 0.93 to $5.22 \mathrm{GPa}$, depending upon the size and spacing of globules. The effective area of adjacent globules over which this stress operates will be presented later in discussions on fracture toughness.

\subsection{Modulus of elasticity of compact hydrated cement paste $\left(E_{o}\right)$}

The modulus of elasticity of compact hcp was determined by considering the effect of relative movement of neighboring C-S-H globules based on the bond force between them. The theoretical cohesive strength between solids is approximated based on the relation between the bond strength $\left(\sigma_{\max }\right)$ between C-S-H globules separated by a distance $\mathrm{d}$ and the corresponding modulus of elasticity $(E)$ and the surface energy $\left(\gamma_{\mathrm{s}}\right)$ as shown in Eq. (2). ${ }^{9}$

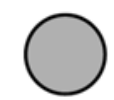

(a) Single C-S-H globule

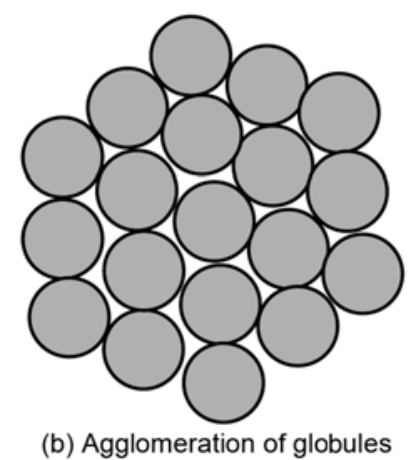

Fig. $1 \mathrm{C}-\mathrm{S}-\mathrm{H}$ morphology and geometric arrangement.

$$
\sigma_{\max }=\sqrt{\frac{E \gamma_{s}}{d}}
$$

This relationship can be reasonably approximated to be about $E_{o} / 10$ for cohesive solids. ${ }^{9}$ Therefore, the relationship between bond strength and the intrinsic modulus of elasticity was approximated as follows:

$$
E_{\mathrm{o}} \approx 10 \sigma_{\max }
$$

where $\sigma_{\max }$ is the bond strength between particles under consideration, that is $F_{v d w}$ (the van der waals force per unit area); the relationship thus becomes:

$$
E_{\mathrm{o}} \approx 10 F_{v d w}
$$

Substituting the values of $F_{v d w}$ derived earlier into Eq. (4) yields the values of $E_{o}$, which range from 9.3 to $52.2 \mathrm{GPa}$ depending upon the size and spacing of C-S-H globules.

\subsection{Fracture toughness of compact hydrated cement paste $\left(G_{0}\right)$}

Fracture toughness of compact hydrated cement paste is defined here as the energy per unit crack area dissipated in the process of breaking C-S-H-C-S-H bonds and pull-out of $\mathrm{CH}$ crystals during fracture. The latter was found to be the major contributor to the fracture toughness (see the following sections). The energy dissipation mechanism in $\mathrm{CH}$ pull-out is related to friction at nanolevel. The frictional stress was thus treated as phononic friction at nano-scale. ${ }^{8}$ The total intrinsic fracture toughness $\left(G_{o}\right)$ was taken as the sum of the energy dissipated due to C-S-H-C-S-H debonding and frictional $\mathrm{CH}$ pull-out; however, the contribution from the former is relatively insignificant.

\subsubsection{Fracture toughness due to C-S-H-C-S-H debonding} $\left(G_{d}\right)$

The fracture toughness contributed by the rupture of interatomic bonds between two C-S-H globules was determined using an equation which relates fracture toughness to van der waals strength as shown below. ${ }^{8}$

$$
F_{v d w}=\left(\frac{3 G_{b}}{2 \pi^{2} k^{2} R_{b}}\right)^{\frac{1}{3}}
$$

where $R_{b}$ is the radius of C-S-H globule (ranges between 3 and 4 $\mathrm{nm}) ; F_{v d w}$ was found earlier to range from 0.93 to $5.22 \mathrm{GPa}$; and

$$
k=\frac{1.5\left(1-v^{2}\right)}{E_{O}}
$$

where $E_{o}$ is intrinsic modulus of elasticity; and $v$ is Poisson's ratio $(\sim 0.2)$ of compact hcp.

Since $R_{b}$ and $F_{v d w}$ are independent variables, a parametric study was conducted to assess the significance of their variation with in viable ranges. The resulting values of $G_{d}$ (fracture toughness due to C-S-H-C-S-H debonding) are presented in Table 1. 
Table 1 Fracture energy due to C-S-H-C-S-H debonding $\left(G_{d}\right)$ in $\mathrm{J} / \mathrm{m}^{2}$

\begin{tabular}{|c|c|c|c|c|c|c|c|c|}
\hline & & \multicolumn{7}{|c|}{$\mathrm{F}_{\mathrm{vdw}}(\mathrm{GPa})$} \\
\hline & & 0.93 & 1.0 & 2.0 & 3.0 & 4.0 & 5.0 & 5.22 \\
\hline \multirow{6}{*}{ 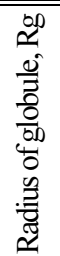 } & 3.0 & 0.38 & "0.41 & 0.82 & 1.23 & 1.64 & 2.05 & 2.14 \\
\hline & 3.2 & 0.41 & 0.44 & 0.87 & 1.31 & 1.75 & 2.18 & 2.28 \\
\hline & 3.4 & 0.43 & 0.46 & 0.93 & 1.39 & 1.86 & 2.32 & 2.42 \\
\hline & 3.6 & 0.46 & 0.49 & 0.98 & 1.47 & 1.96 & 2.46 & 2.56 \\
\hline & 3.8 & 0.48 & 0.52 & 1.04 & 1.56 & 2.07 & 2.59 & 2.71 \\
\hline & 4.0 & 0.51 & 0.55 & 1.09 & 1.64 & 2.18 & 2.73 & 2.85 \\
\hline
\end{tabular}

It can be observed from the table that the maximum contribution of C-S-H-C-S-H debonding to the fracture toughness of compact hcp is $2.85 \mathrm{~J} / \mathrm{m}^{2}$, which is quite small compared to the contribution of $\mathrm{CH}$ pull-out (see next section). Hence, the variations in the C-S-H-C-S-H debonding contributions to fracture toughness are not practically significant.

\subsubsection{Fracture toughness due to $\mathrm{CH}$ Crystal pull-out $\left(\mathrm{G}_{\mathrm{p}}\right)$}

$\mathrm{CH}$ crystals make about $20 \sim 25 \%$ by volume of the hydration product in hcp, ${ }^{10}$ they are generally hexagonal in shape (Fig. 2). ${ }^{11}$ The width of $\mathrm{CH}$ crystals in hcp ranges from 1,000 to $10,000 \mathrm{~nm}$, and their thickness from 220 to $260 \mathrm{~nm} .^{12,13}$ Frictional pull-out of $\mathrm{CH}$ plates from hcp is another factor contributing to the fracture toughness of hcp. For ease of computation, $\mathrm{CH}$ plates were assumed to be circular (instead of hexagonal). The contribution of $\mathrm{CH}$ pull-out to fracture toughness represents the energy dissipated in the form of heat due to phononic friction (associated with atomic vibration) between the surfaces of $\mathrm{CH}$ crystal is the C-S-H nanoparticles. ${ }^{14}$ This frictional energy dissipation during pull-out of $\mathrm{CH}$ crystals depends on the orientation of the $\mathrm{CH}$ crystals relative to the direction of loading. The maximum and minimum energy dissipations occur when the flat surface of $\mathrm{CH}$ crystal is at an angle with and perpendicular to the direction of loading, respectively. Therefore, a vertical $\mathrm{CH}$ orientation, with its flat surface parallel to the direction of loading (Fig. 3), is assumed to account for the random orientation of $\mathrm{CH}$ crystals in hcp.

The portion of the width (diameter) of a single $\mathrm{CH}$ crystal which could be subjected to phononic friction as the $\mathrm{CH}$ pulls out from a cluster of C-S-H globules ranges between 0 and 0.5 (of the width). Therefore, a quarter of the diameter of $\mathrm{CH}$ crystals was assumed to be subject to phononic friction for the purpose of estimating an average $\mathrm{CH}$ pull-out length. The part of $\mathrm{CH}$ crystal

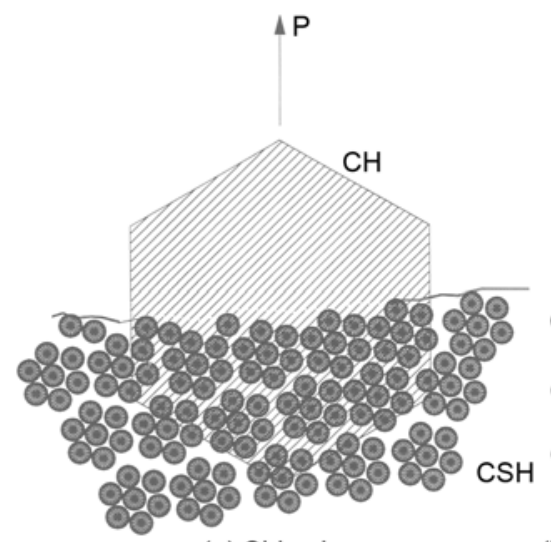

(a) Side view

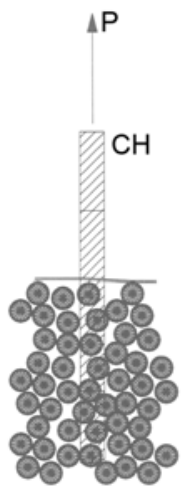

(b) Front view

Fig. $3 \mathrm{CH}$ orientation with flat surface parallel to the direction of loading.

cross-section subjected to frictional pull-out is a segment bound by a chord $0.5 \mathrm{R}$ away from the center of the circle as shown in Fig. 4. The area of this segment $\left(A_{s}\right)$ can be obtained as the difference between the $\mathrm{OABC}$ and the $\mathrm{OAC}$ areas.

$$
A_{s}=2 R^{2} \cos ^{-1}\left(\frac{Z}{R}\right)-Z \sqrt{R^{2}-Z^{2}}
$$

where $R$ is the radius of $\mathrm{CH}$ crystal; and $Z$ is the half of the radius plus the distance moved by $\mathrm{CH}$ as it pulls-out. The value of $Z$ increases during the pull-out process of $\mathrm{CH}$.

The actual contact area between spherical C-S-H globules and the flat surface of circular $\mathrm{CH}$ crystal in compact hydrated cement paste (hcp) was determined based on the assumed morphology

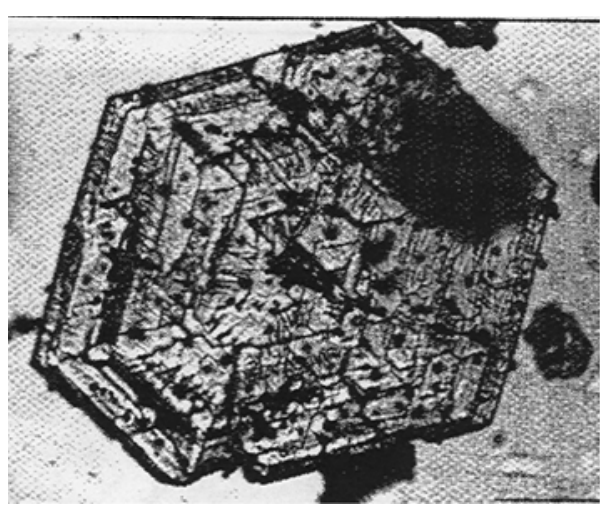

Fig. 2 Hexagonal $\mathrm{CH}$ crystal. $^{11}$

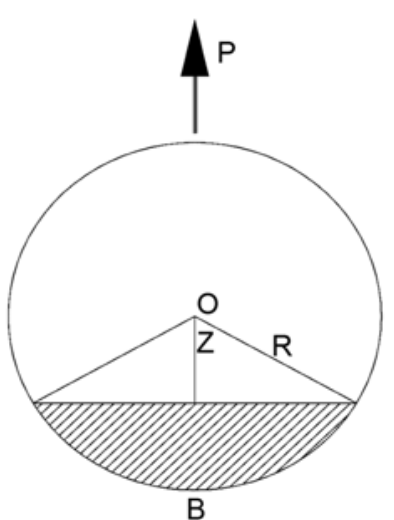

Fig. 4 Schematic model of $\mathrm{CH}$ pull-out. 


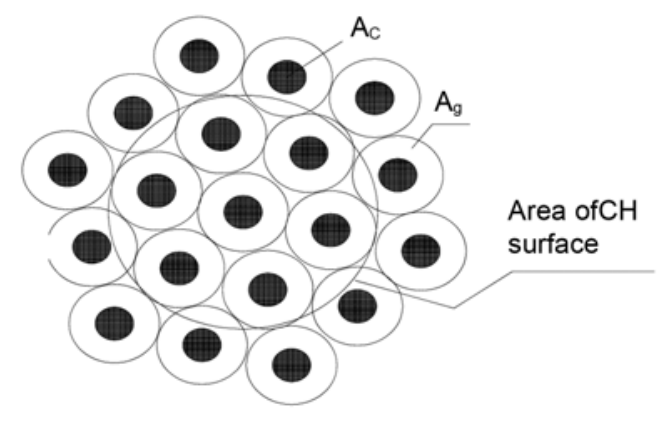

Fig. 5 Schematics of the effective contact area between $\mathrm{CH}$ crystal and $\mathrm{C}-\mathrm{S}-\mathrm{H}$ globules.

and structural arrangement of C-S-H globules (Fig. 5).

The effective contact area $\left(A_{c}\right)$ subjected to bond energy $\left(G_{d}\right)$ per unit area can be determined as follows: ${ }^{8}$

$$
\begin{aligned}
& A_{c}=\pi r_{c}^{2} \\
& r_{c}=\left(1.5 \pi k R_{g}^{2} G_{d}\right)^{1 / 3}
\end{aligned}
$$

where $R_{g}$ is the radius of the C-S-H globule.

The values of $r_{c}$ and $A_{c}$ range from 1.4 to $1.8 \mathrm{~nm}$ and 5.8 to 10 $\mathrm{nm}^{2}$, respectively. The total effective contact area $\left(A_{E C H}\right)$ of all C$\mathrm{S}-\mathrm{H}$ globules contacting both faces of a single $\mathrm{CH}$ crystal can thus be calculated as follows:

$$
A_{E C H}=0.8 \frac{A_{c}}{A_{g}} A_{s}
$$

where $A_{g}$ is the projected area of a C-S-H globule.

The value of $A_{E C H}$ calculated above ranges from 1.11 to $0.00515 \mathrm{~nm}^{2}$. Equation (10) is multiplied by 0.8 to account for the nearly $20 \%$ gel porosity.

The next step was to determine the frictional force acting on the $\mathrm{CH}$ surface as it pulls out. For the atomically sharp surfaces of $\mathrm{CH}$ crystals, nano-scale phononic friction dominates, where collision of vibrating molecules as two flat surfaces slide against each other dissipate energy in the form of heat. The phononic frictional stress $\left(\tau_{f}\right)$ associated with $\mathrm{CH}$ pull-out can be estimated at about 1 GPa. $^{15}$

The frictional force ( $F_{f}$, newtons) due to phononic friction can be computed as the effective area of $\mathrm{CH}$ subjected to frictional pull-out $\left(A_{E C H}, \mathrm{~m}^{2}\right)$ times the phononic friction stress acting on it:

$$
F_{f}=\tau_{f} A_{E C H}=1 \times 10^{9} \times A_{E C H}
$$

The energy dissipated by pull-out of a single $\mathrm{CH}$ crystal, $E_{f 1}$, is computed as the work done by $F_{f}$ during pull-out of $\mathrm{CH}$ crystal over a distance equal to half its radius:

$$
E_{f 1}=\int_{R / 2}^{R} F_{f} d z=\int_{R / 2}^{R} 10^{9} \tau_{f} A_{E C H} d z
$$

The next task is to approximate the possible number of $\mathrm{CH}$ crystals per unit area of hydrated cement paste in order to calculate the amount of energy dissipated per unit cracked area. The number of $\mathrm{CH}$ crystals in a unit cubic cell can be estimated by assuming a uniform distribution of vertically oriented $\mathrm{CH}$ crystals. The amount of $\mathrm{CH}$ in mature Portland cement paste ranges from 20 to 25 percent $^{12}$ of the solid volume (estimated at $22 \%$ on average). The volume of a single $\mathrm{CH}$ crystal $\left(V_{C H}\right)$ can be calculated as:

$$
V_{C H}=\pi R_{C H}^{2} t_{C H}
$$

where $R_{C H}$ and $t_{C H}$ are the radius and thickness of $\mathrm{CH}$ crystal, respectively.

The number of $\mathrm{CH}$ crystals per unit cubic cell volume $\left(N_{C H}\right)$ can be obtained as:

$$
N_{C H V}=\frac{0.22 \times 1}{V_{C H}}
$$

The number of $\mathrm{CH}$ crystals in a plane parallel to any side of the unit cubic cell, $N_{C H P}$, is:

$$
N_{C H P}=\left(N_{C H V}\right)^{2 / 3}
$$

The total energy per unit area $\left(G_{p}\right)$ due to pull-out of $\mathrm{CH}$ crystals can thus be calculated as follows:

$$
G_{p}=E_{f 1} N_{C H P}
$$

The values of $G_{p}$ calculated using the above approach range between 5.42 and $281 \mathrm{~J} / \mathrm{m}^{2}$.

Therefore, the total intrinsic fracture toughness of compact hydrated cement paste $\left(G_{o}\right)$ can be determined as the sum total of energy dissipation by $\mathrm{CH}$ pull-out $\left(G_{p}\right)$ and debonding of C-S-H nanoparticles $\left(G_{d}\right)$ :

$$
G_{o}=G_{d}+G_{p}
$$

The values of $G_{o}$ calculated using the above equation range between 7.55 and $283 \mathrm{~J} / \mathrm{m}^{2}$, compared to measured values range of about 16.9 to $41.0 \mathrm{~J} / \mathrm{m}^{2}$ fracture toughness $\left(G_{I C}\right)$ for compact hydrated cement pastes. ${ }^{16}$

\subsection{Intrinsic tensile strength of compact hydrated cement paste $\left(\sigma_{t o}\right)$}

The tensile strength of compact hcp was determined from the force required to break the bond between two basic C-S-H globules. The total force required to separate two surfaces of physically bonded C-S-H globules was determined as the sum of the forces required to break each bonded C-S-H globule divided by the total debonded surface area.

The tensile force required to break the bond between two basic C-S-H globules $\left(F_{g}\right)$ can be determined as:

$$
F_{g}=A_{c} F_{v d w}
$$

The intrinsic tensile strength $\left(\sigma_{o}\right)$ can be determined, assuming $22 \%$ gel porosity, as follows: 


$$
\sigma_{o}=0.78 \frac{F_{g}}{A_{g}}
$$

The values of $\sigma_{0}$ calculated using the above equations range between 152 and $854 \mathrm{MPa}$, compared with the measured value of about $930 \mathrm{MPa}$ for the tensile strength of compact hcp. ${ }^{4}$

\section{Hydrated cement paste}

\subsection{Introduction}

The term micro-defective hydrated cement paste is used here for a conventional hydrated cement paste (hcp) which incorporates capillary pores and microcracks in its microstructure. The mechanical and physical properties of hcp are affected by the presence of these micro defects, which should be accounted for in calculation of their strength, modulus of elasticity and fracture toughness. Capillary pores and microcracks affect the modulus of elasticity of hydrated cement paste by reducing the effective contact area and generating stress concentration. The shape and size distribution of the capillary pores significantly affect the modulus of elasticity of hcp. Capillary pores are assumed hence to have an elliptical shape with the ratio of the major-to-minor axis about $2 .^{17}$

\subsection{Elastic modulus}

In modeling the elastic modulus of hcp, the first step involves determining the relationship between capillary porosity and pore size. Uniform size capillary pores distributed uniformly in space were assumed for ease of computations (Fig. 6).

If a unit volume of hcp, enclosing a single pore, is considered to have $2 \mathrm{a}+\mathrm{d}$ width, $2 \mathrm{~b}$ thickness and a unit length (see Fig. $6(\mathrm{~b})$ for notation), then porosity can be approximated as:

$$
\begin{aligned}
& p=\frac{\pi a}{4 a+2 d} \\
& \frac{d}{a}=\frac{(\pi-4 p)}{2 p}
\end{aligned}
$$

The effect of interactions between two adjacent capillary pores,

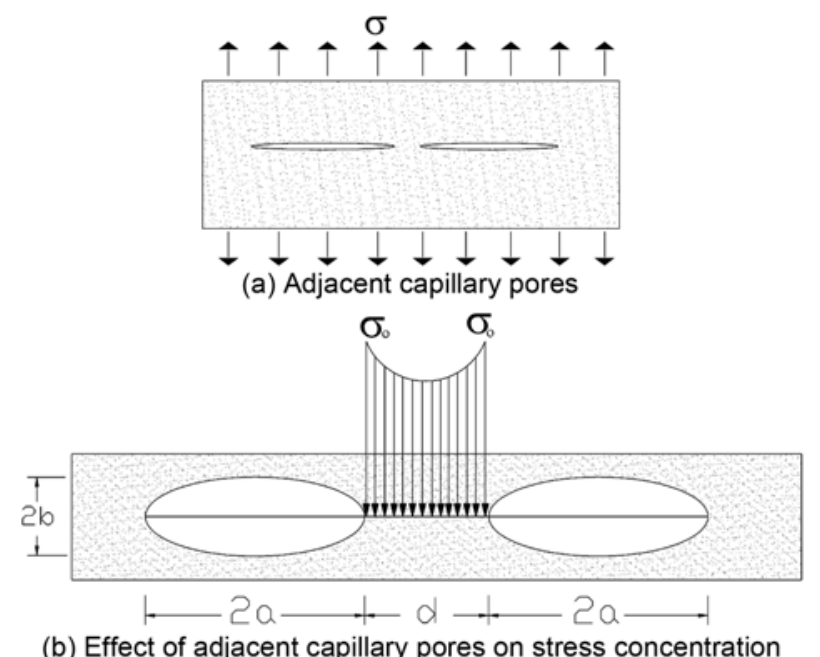

Fig. 6 Capillary pore shape and interactions, and the resulting stress concentration. separated by a distance $\boldsymbol{d}$, on stress concentration in hep can be determined using the relationship between $\boldsymbol{d}$ and the amplification factor $\left(\mathrm{K}_{2}\right)$ with the stress concentration due to a single elliptical capillary pore $\left(\mathrm{K}_{1}\right){ }^{18}$

$$
k_{2}=k_{1}\left(\frac{d}{a}\right)^{-0.37}
$$

where $\mathrm{K}_{1}$ is the stress concentration factor due to a single ellipse; and $\mathrm{K}_{2}$ is the stress concentration amplification factor due to the interaction of adjacent capillary pores. The following expression can be used to calculate $\mathrm{K}_{1}$ :

$$
K_{1}=1+2 \frac{a}{b}
$$

From Fig. 6, it can be observed that $\sigma_{\mathrm{o}}=K_{2} \sigma$, and since for constant strain in the linear elastic range, $E / E_{o}$ is equal to $\sigma / \sigma_{0}$, one concludes that $\mathrm{E} / \mathrm{E}_{\mathrm{o}}=1 / K_{2}$.

$$
\frac{E}{E_{o}}=\frac{\sigma}{\sigma_{o}}=\frac{1}{K_{2}}=\frac{1}{K_{1}}\left(\frac{d}{a}\right)^{0.37}
$$

Substituting Eq. (21) and $\mathrm{K}_{1}$ into Eq. (24), and simplifying the resulting equation, gives the model for the modulus of elasticity of hep,

$$
E=0.77 E_{o} \frac{(1-p)(\pi-4 p)^{0.37}}{\left(1+2 \frac{a}{b}\right) p^{0.37}}
$$

The analytical model is plotted as a function of porosity and compared with empirical models in Fig. 7. The analytical model is observed to compare reasonably well with empirical models which reflect the trends observed in experiments.

\subsection{Fracture toughness of hcp (G)}

The fracture surface of hcp is not highly tortuous, and thus the fracture toughness of hcp $(G)$ can be assumed to vary linearly with porosity as shown in Eq. (26).

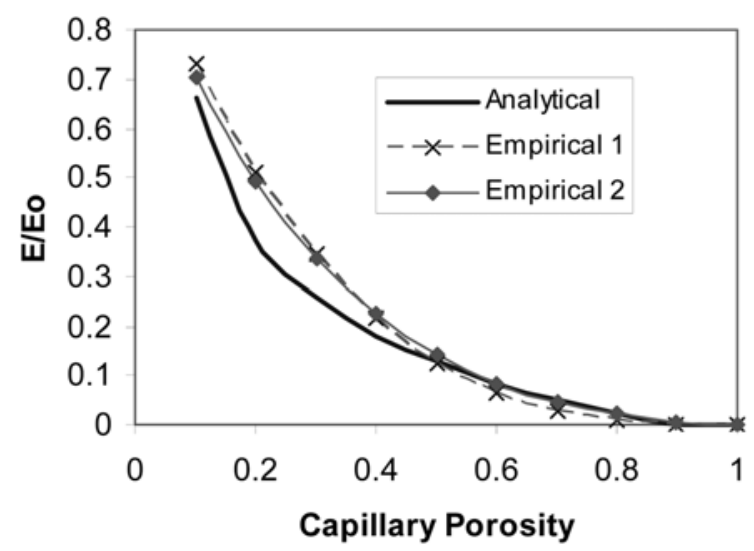

Fig. 7 Modulus of elasticity-porosity relationships. 


$$
G=G_{o}(1-p)
$$

where $G_{o}$ is the fracture toughness of compact hydrated cement paste.

\subsection{Tensile strength of hydrated cement paste}

The Griffith's equation for linear elastic fracture mechanics (LEFM) is used to predict the tensile strength of hep in order to use this model, one needs to determine the critical crack length for hcp. Microcracks develop in cementitious materials even before the application of loads. These microcracks result from the restraint of thermal and drying shrinkage of hcp at early age. Calculation of the critical crack length in hep is presented below.

\subsubsection{Shrinkage microcracks}

The formation of microcracks in hcp is due to the differential shrinkage that exists between the relatively stiff $\mathrm{CH}$ crystal and the softer C-S-H gel. Restraint of the shrinkage strains in C-S-H gel by $\mathrm{CH}$ crystals lead to microcracking of the young hcp. Restrained shear stresses cause debonding of C-S-H from the $\mathrm{CH}$ surface and subsequent cracking of hcp (Fig. 8).

The strain energy released during cracking under the effect of shrinkage strain can be calculated as follows:

$$
U_{e}=\varepsilon_{s h} E_{o} A y
$$

where $\varepsilon_{s h}=$ shrinkage strain; $E=$ modulus of elasticity of C-S-H; $A$ (surface area of $\mathrm{CH}$ subjected to skin friction) $=\left(P_{C H}\right)\left(t_{C H}\right) ; \mathrm{y}$ (crack width at the periphery of $\mathrm{CH}$ crystal) $=\varepsilon_{s h} P_{C H}$; and $P_{C H}$ and $\mathrm{t}_{\mathrm{CH}}$ are the perimeter and thickness of $\mathrm{CH}$ crystal, respectively.

Substituting the above expressions into Eq. (27):

$$
U_{e}=\left(\varepsilon_{s h} P_{C H}\right)^{2} E_{o} t_{C H}
$$

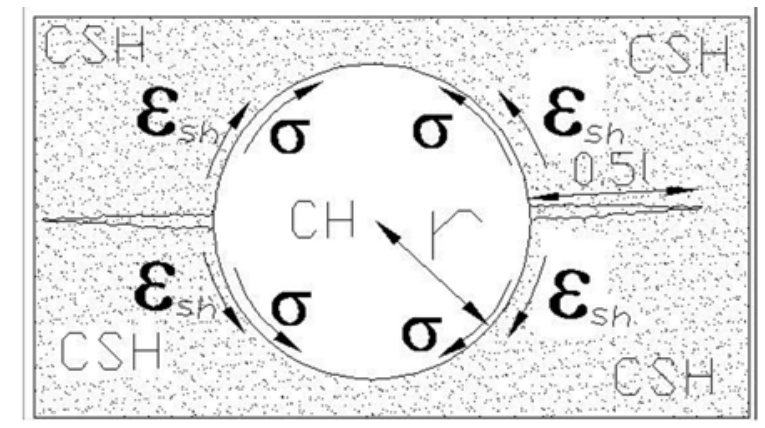

(a) Shrinkage cracking around $\mathrm{CH}$ crystal

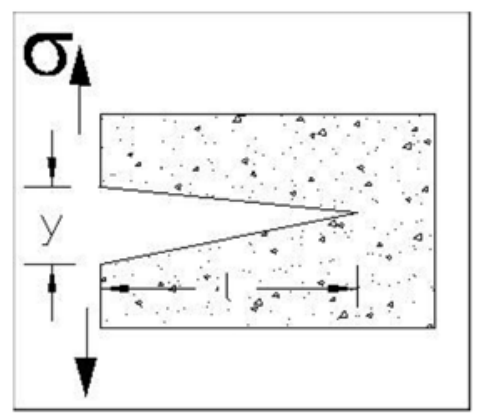

(b) Simplified crack model

Fig. 8 Schematic presentation of the formation of microcracks in otherwise micro-defect-free hcp.
The energy dissipated upon cracking $\left(U_{i}\right)$ can be calculated as follows:

$$
U_{i}=G_{d} l t_{C H}
$$

where $G_{d}$ is the fracture toughness due to C-S-H debonding; and $l$ is crack length.

Equating $\mathrm{U}_{\mathrm{e}}$ (Eq. 28) and $\mathrm{U}_{\mathrm{i}}$ (Eq. (29)) yields the following expression for crack length, $l$ :

$$
l=\frac{\left(\varepsilon_{s h} P_{C H}\right)^{2} E_{o}}{G_{d}}
$$

Due to the unavailability of sufficient information on the relationship between capillary porosity $(p)$ and shrinkage strain $\left(\varepsilon_{s h}\right)$, an empirical relationship was determined using experimental results $^{12}$ as follows:

$$
\varepsilon_{s h}=0.0148 p-8.8 \times 10^{-4}
$$

After substituting Eq. (31) into Eq. (30) and simplifying it, the following expression can be derived for crack length:

$$
l=\frac{39.8 E_{o} r_{C H}^{2}\left(0.0148 p-8.8 \times 10^{-4}\right)^{2}}{G_{d}}
$$

where $r_{C H}$ is the radius of the largest possible $\mathrm{CH}$ crystal; a 100 $\mu \mathrm{m}$ diameter $\mathrm{CH}$ was considered. ${ }^{12}$ The critical crack length $l^{\prime}$ is half of $l$ plus the diameter of $\mathrm{CH}$ :

$$
l^{\prime}=0.5\left(1+2 r_{C H}\right)
$$

\subsubsection{Tensile strength of hydrated cement paste}

The Griffith equation defines the relationship between tensile strength $\left(\sigma_{t}\right)$ and critical crack length $\left(l^{\prime}\right)$ :

$$
\sigma_{t}=\sqrt{\frac{E G}{\pi l^{\prime}}}
$$

Substituting Eq. (25) for E, Eq. for G and Eq. (33) for $l$ ' into Eq. (34) yields:

$$
\sigma_{t}=\sqrt{\frac{0.77 E_{o} G_{o}(1-p)^{2}(\pi-4 p)^{0.37}}{\pi l^{\prime}\left(1+2 \frac{a}{b}\right) p^{0.37}}}
$$

\section{Validation of tensile strength model of hcp}

The values of different parameters governing the tensile strength of hcp are determined using the ranges presented in the literature (presented earlier), with parametric studies conducted to assess the significance of various parameters in order to assist with final values within the reported ranges. The final selections were as follows: $r_{C H}$ (radius of largest possible $\mathrm{CH}$ crystal) $=50,000$ $\mathrm{nm} ; E_{o}=38 \mathrm{Gpa} ; G_{d}=1.56 \mathrm{~J} / \mathrm{m}^{2}$; and the ratio of the major-tominor axis of capillary pores was $2 .^{17} E_{o}$ and $G_{d}$ were calculated 


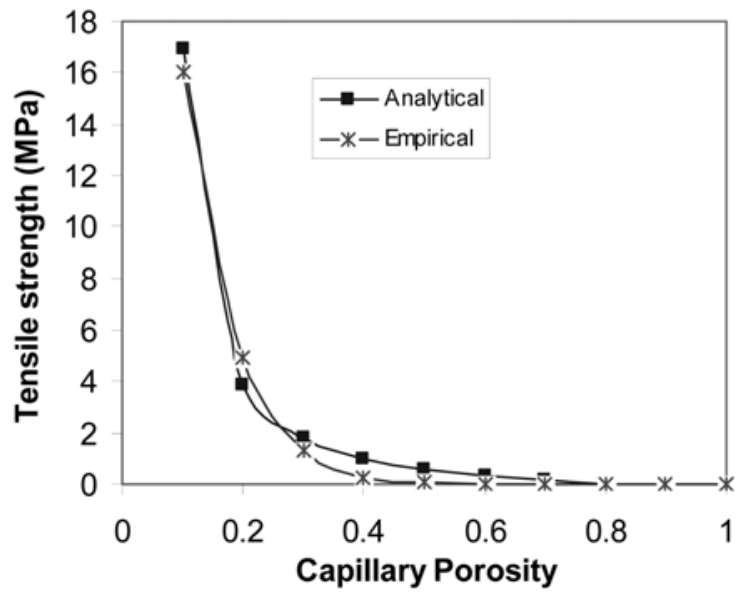

Fig. 9 Influence of the modified range of parameters on the strength model of hcp.

by considering the average values of $\mathrm{d}$ (the distance between two C-S-H globules) and $R_{b}$ (the radius of C-S-H globule) to be 0.2 $\mathrm{nm}$ and $3.5 \mathrm{~nm}$, respectively. ${ }^{6}$ Average thickness and diameter of $\mathrm{CH}$ crystal were assumed to be $240 \mathrm{~nm}$ and 2,500 nm, respectively. Substituting the above values into Eq. (37) yields:

$$
\sigma_{t}=6726(1-p) \sqrt{\frac{(\pi-4 p)^{0.37}}{p^{0.37}\left(0.0148 p-8.8 \times 10^{-4}\right)^{2}+41.3}}
$$

The above equation is observed in Fig. 9 to compare favorably with an empirical model which reflects experimental result.

\section{Conclusions}

The following conclusions can be drawn based on the results obtained from the above work:

1) The mechanical properties and mode of failure of hydrated cement paste at nano-scale depend on bond strength between C-S$\mathrm{H}$ globules resulting from the interatomic interactions. However, the introduction of capillary pores and microcracks at micro- and macro-scales alters the property and modes of failures. In the former, failure is governed by debonding of C-S-H globules while in the later by fracture.

2) It can be observed from the research that fracture toughness of hydrated cement paste depends on two energy dissipation mechanisms, debonding of C-S-H globules and $\mathrm{CH}$ pull-out friction. The later is the major contributor to the fracture toughness of hydrated cement paste.

3) These mechanical models can be used as a basis to promote further researches aiming at developing models for various cementitious products incorporating different mineral admixtures.

\section{References}

1. Balshin, M. Y. "Relation of Mechanical Properties of Powder Metals and Their Porosity and the Ultimate Properties of Porous Metal-ceramic Materials" Dokl. Akad. Nauk. (SSSR), 67, No. 5, 1949, pp. 831 834.

2. Ryshkewitch, R., "Compression Strength of Porous Sin- tered Alumina and Zirconia," Journal of American Ceramics Society, Vol. 36, 1953, pp. 65 68.

3. Jonsson, B., Wennerstrom, H., Nonat, A., and Cabane B., "Onset of Cohesion in Cement Paste," Langmuir, Vol. 20, 2004, pp. 6702 6709.

4. Plassard, C., Lesniewska, E., Pochard, I., and Nonat, A., "Nanoscale Experimental Investigation of Particle Interactions at the Origin of the Cohesion of Cement," Langmuir, Vol. 21, 2005, pp. 7263 7270.

5. Richardson, I. G., "Tobermorite/Jennite-and Tobermorite/ Calcium Hydroxide-Based Models for the Stricter of C-S-H: Applicability to Hardened Pastes of Tricalcium Silicate, BDicalcium Silicate, Portland Cement, and Blends of Portland Cement With Blast-Furnace Slag, Metakaolin, or Silica Fume," Journal of Cement Concrete Research, Vol. 34, 2004, pp. 1733 1777.

6. Jennings, H. M., "A Model for the Microstructure of Calcium Silicate Hydrate in Cement Paste," Journal of Cement Concrete Research, Vol. 30, 2000, pp. 101 116.

7. Thomas, J. J. and Jennings. H. M., "A Colloidal Interpretation of Chemical Aging of the C-S-H Gel and its Effects on the Properties of Cement Paste," Journal of Cement Concrete Research, Vol. 36, 2006, pp. 30 38.

8. Persson, B. N. J., Sliding Friction: Physical Principles and Applications, Germany: Springer (Nanoscience and Technology), 1998, pp. 54 59.

9. Felbeck, D. K. and Atkins, A. G., Strength and Fracture of Engineering Solids, New Jersey, Prentice-Hall, 1984.

10. Harutyunyan, V. S., Abovyan, E. S., Monterio, P. J. M., Mkrtchyan, V. P., and Balyan, M. K., "Microstrain Distribution in Calcium Hydroxide Present in the Interfacial Transition Zone," Journal of Cement Concrete Research, Vol. 30, 2000, pp. 709 713.

11. Barker, A. P., "Structural and Mechanical Characterization of Calcium Hydroxide in Set Cement and the Influence of Various Additives," World Cement. Vol. 15, 1984, pp. 25 28.

12. Mindess, S., Young, J. F., and Darwin, D., Concrete, New Jersey: Prentice-Hall, 2003.

13. Diamond, S. and Bonen, D., "Microstructure of Hardened Cement Paste - A New Interpretation," Journal of American Ceramic Society, Vol. 76, 1993, pp. 2993 2999.

14. Popov, V. L., "Electronic and Phononic Friction of Solids at Low Temperatures," Tribology International, Vol. 34, 2001, pp. 277 286.

15. Meyer, E., Overney, R. M., Dransfeld, K., and Gyalog, T., Nanoscience: Friction and Rheology on the Nanometer Scale, Singapore: Eurasia Press, 1998.

16. Alford, N. M., Groves, G. W. and Double, D. D., "Physical Properties of High Strength Cement Pastes," Journal of Cement Concrete Research, Vol. 12, 1982, pp. 349 358.

17. Ammouche, A., Breysse, D., Hornain, H., Didry, O. and Marchand, J., "A New Image Analysis Technique for the Quantitative Assessment of Microcracks in Cement-Based Materials," Cement and Concrete Research, Vol. 30, 2000, pp. 25-35.

18. Tsukrov, I. and Kachanov, M., "Stress Concentrations and Microfracturing Patterns in a Brittle-Elastic Solid with Interacting Pores of Diverse Shapes," International Journal of Solid Structures, Vol. 34, 1997, pp. 2887 2904. 